\title{
Nicht-invasive, online Dichtebestimmung mittels ultraschallbasierender Mehrfach-Reflektions-Methode
}

\author{
S. Hoche ${ }^{1}$, M.A. Hussein ${ }^{1}$, T. Becker ${ }^{1}$ \\ ${ }^{1}$ Technische Universität München, Lehrstuhl für Brau- und Getränketechnologie, (Bio-) Prozess \\ Analyse and Prozess Technologie, Weihenstephaner Steig 20, 85354 Freising, Germany, \\ hussein@wzw.tum.de
}

\begin{abstract}
Die Dichte ist ein wichtiger Parameter für die meisten bekannten Verfahren der chemischen, petrochemischen, pharmazeutischen, Lebensmittel- und Getränkeindustrie; sowie Biotechnologie, Wasser- und Abwasserwirtschaft. Das Potenzial den Prozess online mittels Dichte zu beurteilen, ermöglicht neue Möglichkeiten der Prozesssteuerung und -überwachung. Bei einigen Methoden werden direkte physikalischen Beziehungen genutzt, die letztendlich auf der Korrelation zwischen Dichte und Prozessgröße basieren und auf bestimmte chemische Reaktionen oder charakteristische Prozessverläufe beschränkt sind. Etablierte Messsysteme wie Coriolis-Masse- oder SchwingrohrSysteme sind wiederum oftmals auf spezielle Anwendungsbereiche (z.B. bezüglich Sensorimplementierung: Rohrdurchmesser, Bypass, Durchflussgeschwindigkeit) beschränkt. Je nach Prozess können weitere Limitierungen wie Empfindlichkeit gegenüber Blasen, Partikel oder Fouling relevant sein. In sensitiven Bereichen wie der Lebensmittel- oder pharmazeutischen Industrie sind zusätzlich Aspekte des Hygienic Design und Sterilisierbarkeit von besonderer Bedeutung. Dieser Beitrag befasst sich mit der alternativen, nicht-invasiven, online Bestimmung der Dichte mittels ultraschallbasierender Mehrfach-Reflektions-Methode und Dämpfer-Reflektor-Sonde. Kalibrationsversuche ergaben einen absoluten, mittleren, quadratischen Fehler von $\pm 0,5 \mathrm{~kg} / \mathrm{m}^{3}$. Evaluationsversuche in der kontinuierlichen Kultur ergaben einen mittleren Fehler von 0,77 \%.
\end{abstract}

Key words: Prozessüberwachung, Fermentation, Ultraschall, Reflektionskoeffizient, Dichte

\section{Einleitung}

In der Vergangenheit wurden verschiedene Methoden der ultraschallbasierenden Dichtebestimmung untersucht [1]. Vielversprechende Methoden die Dichte von Multi-Komponenten-Gemischen zu bestimmen, sind die sogenannten Dämpfer-Methoden (BRTs). Grundlage dieser Methoden sind die ebene Wellenausbreitung über eine oder mehrere Phasengrenzflächen und das Wissen der temperaturabhängigen Eigenschaften des Dämpfermaterials. Die BRTs können grob in vier Untergruppen unterteilt werrden: die Mehrfachreflektionsmethoden (MRM), die Transmissionsmethoden (TM), die Referenzmethoden (RRM) und die Winkelreflektionsmethoden (ARM). Je nach Anwendungsfall ergeben sich für die Untergruppen verschiedene Vor- und Nachteile. Im Falle einer Anwendung in der Getränkeindustrie ist mit moderater Absorption, variablen Prozessbedingungen und Temperaturgradienten zu rechnen. Unter Berücksichtigung der prozessbezogenen und konstruktiven Randbedingungen in Relation zu den Analysemöglichkeiten, dem geringen Kalibrationsaufwand sowie den anfallenden Kosten ist die MRM die beste Lösung.

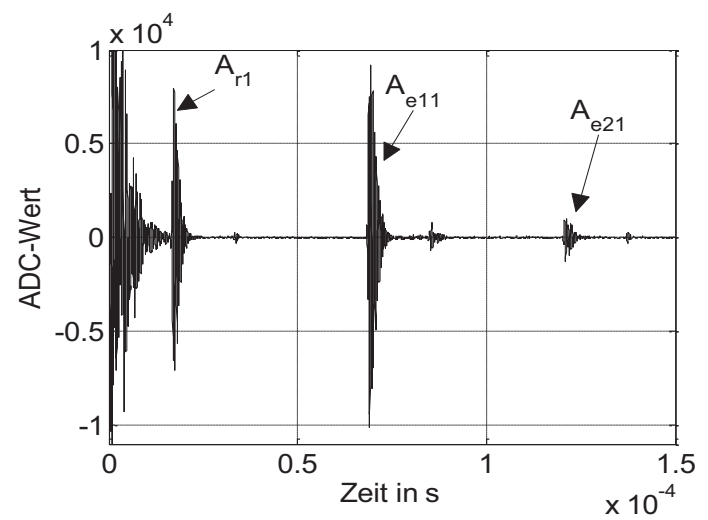

Abb. 1. Vollständiges, mittels des beschriebenen Sensors aufgezeichnetes Signal

Zunächst wird der Reflektionskoeffizient (RC) und die Signallaufzeit (TOF) bestimmt. Dazu müssen die Amplituden von drei relevanten Nutzsignalen ausgewertet werden: $A_{r 1}, A_{e 11}$ and $A_{e 21}$ (siehe Abb. 1). 
Detailliertere Informationen bezüglich der Methode sind in [1-6] zu finden. Sind die relevanten Amplituden (A) bestimmt, kann der Reflektionskoeffizient von Medium 1 zu Medium 2, $r_{12}$ wie folgt berechnet werden:

$r_{12}=\sqrt{\frac{x}{x-1}}$ wobei $x=\frac{A_{r 1} \cdot A_{e 21}}{A_{e 11}{ }^{2}}$.

Die Indices der Amplituden beschreibt dabei die Position der einzelnen Nutzsignale innerhalb des Gesamtsignals (siehe Abb. 1); nicht zu verwechseln mit den Indices des Reflektionskoeffizienten, $\mathrm{r}_{\mathrm{km}}$; hier beschreibt $\mathrm{k}$ das Material der Einfallsseite und $m$ das Material in Transmissionsrichtung. Die Materialien sind wie folgt aufgeschlüsselt: 1 Dämpfermaterial, 2 - Probenflüssigkeit, 3 Reflektormaterial. $\mathrm{TOF}_{2}$ wurde aus der Differenz der Einzelsignalzeiten berechnet:

$\mathrm{TOF}_{2}=t_{e 11}-t_{r 1}$

wobei die Zeiten der Einzelsignale $A_{r 1}$ und $A_{e 11}$ mittels polynomisch approximierter Nulldurchgangsbestimmung bestimmt wurden. Die Ultraschallgeschwindigkeit der Probenflüssigkeit kann wiederum mittels des bekannten Abstands, $I_{2}$ des Reflektors zum Dämpfer berechnet

$$
U S V_{2}=2 \cdot l_{2} / \mathrm{TOF}_{2} \text {. }
$$

Die temperaturabhängige Dichte, $\rho_{1}$ und Ultraschallgeschwindigkeit, $\quad \mathrm{USV}_{1}$ des Dämpfermaterials sind bekannt. Somit kann die Dichte der Probenflüssigkeit, $\rho_{2}$ bei aktueller Temperatur berechnet werden:

$$
\rho_{2}=\frac{\rho_{1} \cdot U S V_{1}}{U S V_{2}} \cdot \frac{\left(1+r_{12}\right)}{\left(1-r_{12}\right)} .
$$

\section{Material und Methoden}

Auf Grundlage bekannter Materialdaten und physikalischer Gesetzmäßigkeiten wurde ein Sensor für den Ingold-Prozesszugang entworfen. Der Dämpfer besteht aus ca. $20 \mathrm{~mm}$ dickem PMMA; der Reflektor aus Edelstahl, wobei der Abstand zum Dämpfer ca. $25 \mathrm{~mm}$ beträgt. Der exakte Abstand und dessen Temperaturabhängigkeit wurde über eine Kalibration mit destilliertem Wasser ermittelt [7]. Die Ultraschallwellen wurden mittels eines PMN-Transducers (PMN: Bleimetaniobat, $2 \mathrm{MHz}, \varnothing 10 \mathrm{~mm}$ ) erzeugt und aufgezeichnet. Die Evaluationsversuche erfolgten in einem Braun $®$ Laborfermenter (B Braun ${ }^{\circledR}$ Biostat UD30). Es wurde Malzextrakt (Fi. Weyermann) mit dem Hefestamm Saccharomyces cerevisiae fermentiert.

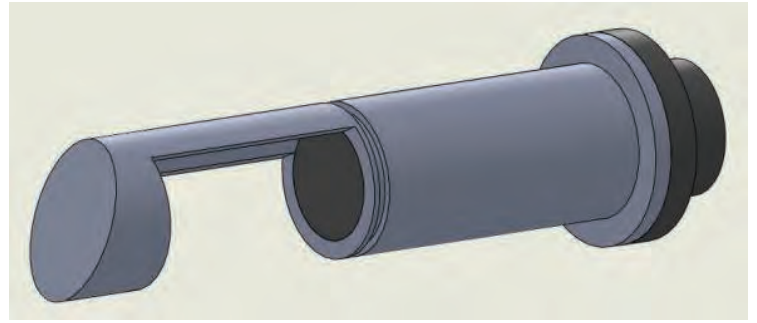

Abb. 2. 3-D-Modell des verwendeten Sensors

Um die Zielhefezellzahl zu erreichen, wurde zunächst aerob fermentiert. In dem für die Evaluation relevanten Zeitbereich, wurde die Fermentation anaerob nach dem ChemostatPrinzip durchgeführt. Die Referenzanalysen wurden mittels Alcolyzer Beer Analyzing System (DMA 4500 M, Anton Paar) ausgewertet.

\section{Resultate und Diskussion}

Die Kalibrationsversuche mit demineralisiertem Wasser ergaben einen absoluten, mittleren, quadratischen Fehler von $\pm 0,5 \mathrm{~kg} / \mathrm{m}^{3}$ (siehe Abb. 3).

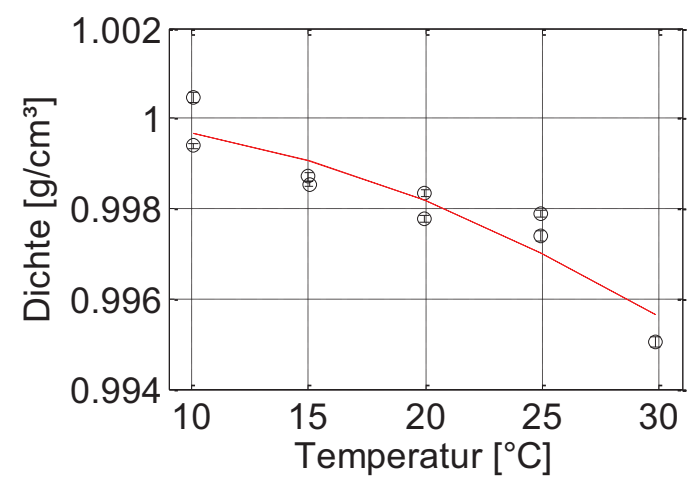

Abb. 3. Ergebnisse der Validierung der Prozesssonde mit destilliertem Wasser und LDens313 Dichtemessgerät als Referenz.

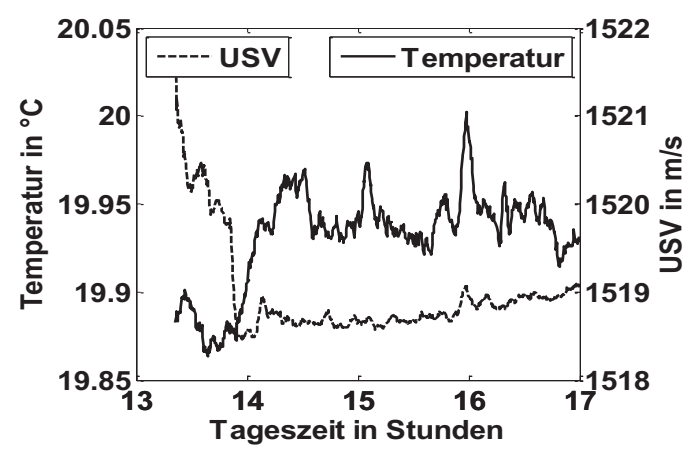

Abb. 4. USV- und Temperatur-Resultate der Evaluation des beschriebenen Sensors am Laborfermenter (Chemostat). 
Die Resultate der Evaluation im Laborfermenter sind in den Abbildungen 4, 5 \& 6 dargestellt. Basierend auf den Referenzdaten der Laboranalysen und den Prozessdaten kann im Zeitraum 14-16 Uhr von ausreichend konstanten Konzentrations-verhältnissen entsprechend der Chemostat-Prozessführung ausgegangen werden.

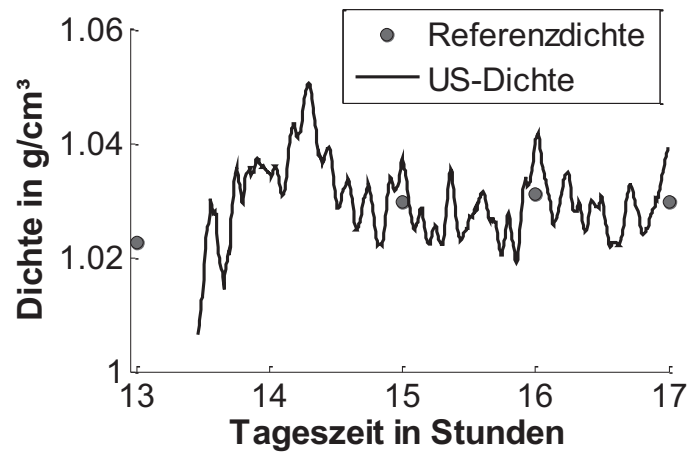

Abb. 5. Dichte-Resultate der Evaluation des beschriebenen Sensors am Laborfermenter (Chemostat).

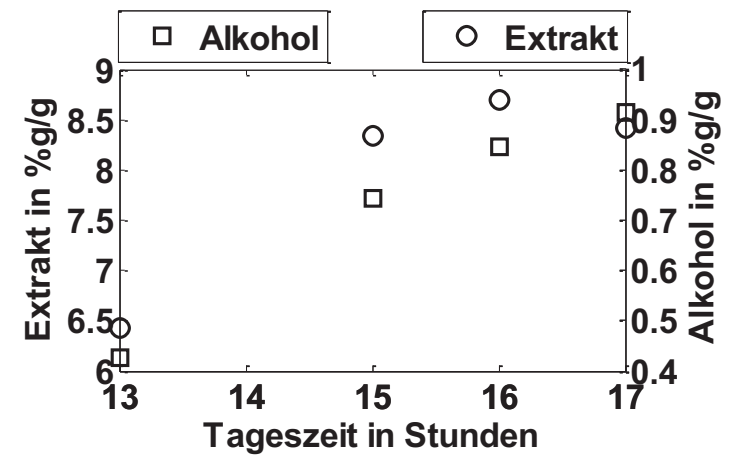

Abb. 6. Dichte-Resultate der Evaluation des beschriebenen Sensors am Laborfermenter (Chemostat).

Der mittlere, quadratische Fehler der ultraschallbasierenden Dichte (siehe Abb. 5) bezüglich der erwarteten Dichte ensprechend der beobachteten Temperatur und Konzentrationsverhältnisse beträgt 0,77\%. Inwiefern die beobachteten Dichteschwankungen auf tatsächliche Schwankungen aufgrund der kontinuierlichen Zufuhr von Nährmedium und Entnahme vom Produkt zurückzuführen sind, kann mangels eines online Referenzsensors nicht beurteilt werden.

\section{Danksagung}

Die Forschungsergebnisse entstanden im Rahmen des Forschungsprojektes $16536 \mathrm{~N}$ und wurden über die AiF und die WiFö (Wissenschaftsförderung der Deutschen Brauwirtschaft e.V., Berlin) im Rahmen des Programms zur Förderung der Industriellen Gemeinschaftsforschung (IGF) vom
Bundesministerium für Wirtschaft und Technologie aufgrund eines Beschlusses des Deutschen Bundestages gefördert.

\section{Literaturangaben}

[1] S. Hoche, M.A. Hussein, T. Becker, Ultrasound-based density determination via buffer rod techniques: a review, J. Sens. Sens. Syst. 2013, 2, 103-125.

[2] E.P. Papadakis, Buffer-Rod System for Ultrasonic Attenuation Measurements, The Journal of the Acoustical Society of America 1968, 44(5), 1437-1441.

[3] J.C. Adamowski, C. Buiochi, C. Simon, E.C.N. Silva, et al., Ultrasonic measurement of density of liquids, $J$. Acoust. Soc. Am. 1995, 97(1), 354-361.

[4] R.T. Higuti, F.R. Montero de Espinosa, J.C. Adamowski, Energy method to calculate the density of liquids using ultrasonic reflection techniques, Proc. IEEE Ultrason. Symp. 2001, 319-322.

[5] E. Bjørndal, K.E. Frøysa, Acoustic Methods for Obtaining the Pressure Reflection Coefficient from a Buffer Rod Based Measurement Cell, IEEE Trans UFFC 2008, 55(8), 1781-1793.

[6] E. Bjørndal, K.E. Frøysa, S.A. Engeseth, A Novel Approach to Acoustic Liquid Density Measurements Using a Buffer Rod Based Measuring Cell, IEEE Trans UFFC 2008, 55(8), 1794-1808.

[7] W. Marczak, Water as standard in the measurements of speed of sound in liquids, J. Acoust. Soc. Am. 1997, 102, 2776-2779. 